\title{
Sur quelques Mesostepbanus Lutz, 1935 (Strigeata : Cyathocotyloidea)
}

\author{
par G. DUBOIS \\ Grand-Rue 12, CH-2035 Corcelles.
}

\section{Résumé.}

Huit espèces de Mesostephanus sont revues ou redécrites. Une nouvelle diagnose générique est proposée.

\section{Summary.}

About several Mesostephanus Lutz, 1935 (Strigeata, Cyathocotyloidea).

Eight species of Mesostephanus are revised or redescribed. A new generic diagnosis is proposed.

\section{Introduction}

Dans l'état actuel de nos connaissances, le genre Mesostephanus Lutz, 1935, serait représenté par une quinzaine d'espèces, après élimination de celles qui sont transférées dans les taxons Prohemistomum Odhner, 1913 (1) (M. indicum Mehra, 1947, syn. $M$. lutzi Vidyarthi, 1948), Neogogatea Chandler et Rausch, 1947 [M. kentuckiensis (Cable, 1935) Myer, 1960] et Mesostephanoides Dubois, 1951 (M. burmanicus Chatterji, 1940)

Ce sont :

* Mesostephanus alopicis Malczewski, 1964.

Mesostephanus appendiculatoides (Price, 1934) Lutz, 1935.

Mesostephanus appendiculatus (Ciurea, 1916) Lutz, 1935.

(1) Dans le genre Prohemistomum, la partie terminale de l'utérus est différenciée en metraterm : il n'y a pas de sphincter vaginal (organe très caractéristique de Mesostephanus). Le metraterm de $P$. indicum (Mehra, 1947) mesure environ $130 \mu$ de longueur. 


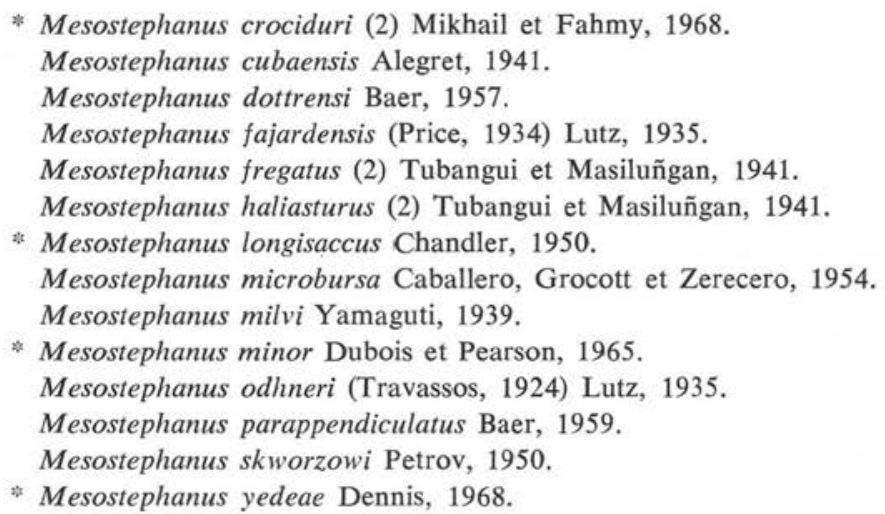

Nous avons eu l'occasion d'examiner les matériaux de plusieurs de ces espèces, ce qui nous a permis de confirmer le statut de la plupart d'entre elles et d'en éliminer quelques-unes comme synonymes (marquées d'un *).

Nous remercions ici le $\mathrm{P}^{\mathrm{r}}$ Jean G. Baer, de l'Université de Neuchâtel, du prêt de ses préparations de Mesostephanus dottrensi; le $\mathrm{D}^{r} \mathrm{I}$. Pérez Vigueras, de l'Université de La Havane, de nous avoir remis le matériel original de Mesostephanus cubaensis Alegret ; le $\mathrm{D}^{r}$ Robert F. Hutton, anciennement au State Board of Conservation Marine Laboratory de St Petersburg, Fla., du don d'une préparation de $M$. appendiculatoides (Price) ; le $\mathrm{D}^{\mathrm{r}}$ Ralph Lichtenfels, de l'U.S. National Museum, à Beltsville, Md., du prêt de l'holotype de $M$. longisaccus Chandler et de l'exemplaire du \& $M$. appendiculatoides (Price) » déposé dans ce musée par Cable, Connor et Balling, et que nous avons identifié avec $M$. odhneri (Travassos). Nous sommes redevable du prêt des nombreuses préparations de cette dernière espèce, que feu le $\mathrm{D}^{\mathrm{r}} \mathrm{J}$. F. Teixeira de Freitas nous envoya de l'Institut Oswaldo Cruz, Rio de Janeiro. Enfin, nous remercions le $\mathrm{P}^{r}$ A. Chabaud, du Museum national d'Histoire naturelle, à Paris, le $\mathrm{P}^{\mathrm{r}}$ Claude Vaucher, de l'Université de Neuchâtel, et le $\mathrm{D}^{r} \mathrm{~B}$. Hörnung, de l'Institut für Tierpathologie, à Berne, de nous avoir fourni des photocopies de diverses publications.

\section{Mesostephanus appendiculatoides (Price, 1934) Lutz, 1935}

Syn. (?) Mesostephanus yedeae Dennis, 1968.

Cette espèce, qui peut atteindre $1,3 \mathrm{~mm}$ de longueur, est caractérisée par la petitesse de la ventouse ventrale faiblement musculeuse, par un court appendice dorsosubterminal (140-150 $\mu$ de longueur sur 200-260 $\mu$ de diamètre à la base), par une poche du cirre longuement claviforme, qui atteint la zone du testicule antérieur et dont la vésicule séminale occupe les deux cinquièmes, par la situation de l'ovaire au niveau intertesticulaire ou du bord postérieur du premier testicule. Elle a été décrite par

(2) L'orthographe correcte devrait être crocidurae, fregatae et haliasturis. 
E. W. Price, d'après un matériel provenant d'un Pelecanus occidentalis L., de la République Dominicaine, puis retrouvée par Caballero, Grocott et Zerecero (1953) en République de Panama, chez Pelecanus occidentalis californicus Ridgway, recueillie encore par Hutton et Sogandares-Bernal (1960 a) en Floride, chez Pelecanus occidentalis carolinensis Gm. (3), enfin signalée par Charles H. Courtney, M.S. Cand. de l'University of Florida Gainesville (coll. 23 mai 1972, à Bird Key, Lee Co., et 12 septembre 1973, à Cocoa Beach), également en Floride, chez ce dernier hôte.

Hutton et Sogandares-Bernal $(1960 \mathrm{~b})$ ont décrit le cycle vital de ce Mesostephanus, dont la cercaire se développe chez Cerithium muscarum Say.

Il est vraisemblable que le Mesostephanus yedeae Dennis, 1968 (non décrit au stade adulte), soit identifiable avec $M$. appendiculatoides. Ce Ver, dont Dennis a réalisé le cycle vital, est parasite de Pelecanus occidentalis L., en Floride, et sa cercaire se développe chez Cerithium literatum Born.

Le «Mesostephanus appendiculatoides», dont Cable, Connor et Balling (1960, pp. 197-198, fig. 5) ont recueilli deux petits exemplaires ovigères chez une Sula leucogaster (Bodd.) à Porto Rico, le 15 juillet 1952, doit s'identifier plutôt avec le Mesostephanus odhneri (Travassos, 1924) retrouvé au Brésil chez Sula sula (L.) et Fregata aquila (L.) (coll. A. Lutz de l'Institut Oswaldo Cruz, Rio de Janeiro). La description des auteurs américains correspond à celle que Dubois (1970, pp. 192-193, fig. 1 et 2) a donnée de l'espèce de Travassos, notamment en ce qui concerne la forme du corps, le rapport dimensionnel des ventouses, l'égalité de longueur de la ventouse buccale et du pharynx, la situation de l'acetabulum, la disposition des vitellogènes, la localisation de l'ovaire à la hauteur du premier testicule, l'extension de la poche du cirre longuement claviforme, qui peut atteindre le bord frontal de ce dernier, la subégalité de longueur de la vésicule séminale et de la pars prostatica (vide infra: $M$. odhneri).

\section{Mesostephanus appendiculatus (Ciurea, 1916) Lutz, 1935}

Syn. Mesostephanus longisaccus Chandler, 1950.

Mesostephanus appendiculatus, décrit par Ciurea comme parasite de l'intestin grêle de trois chiens et d'un chat nourris de différents poissons du Danube, est caractérisé par un pharynx ellipsoïdal bien développé, très musculeux et presque aussi grand que la ventouse buccale, par un œsophage court (25-45/20-25 $\mu$ ) (4), également musculeux, apparaissant comme un manchon à fibres transversales très visibles qui délimitent une lumière étroite, par une ventouse ventrale subégale à la ventouse buccale ou plus petite, et par une poche du cirre atteignant le milieu de la zone du testicule antérieur. W. E. Martin (1961), qui a réalisé expérimentalement le cycle vital de

(3) Hutton et Sogandares-Bernal $(1960 \mathrm{~b})$ ont obtenu ce ver chez un Nycticorax nycticorax hoactli (Gm.), un Larus delawarensis Ord. et un Procyon lotor (L.) infestés expérimentalement par de la viande de Muge (Mugil). Hutton (1964) cite encore comme hôtes expérimentaux Didelphis marsupialis L., Procyon lotor (L.) et Casmerodius albus egretta (Gm.).

(4) Ciurea indique : "Immédiatement derrière le pharynx se produit la bifurcation intestinale. » 
M. appendiculatus en Californie (5), précise que l'œsophage est «approximately onehalf pharyngeal length, with transverse muscle fibers » (voir sa figure 1). Les œufs mesurent 91-117/62-68 $\mu$ (d'après Dubois, 1938), 84-137/58-81 $\mu$, moyenne 108/70 $\mu$. (d'après Martin).

Ces caractères se retrouvent dans l'holotype de Mesostephanus longisaccus Chandler (U.S. Nat. Mus., Helm. Coll., $\mathrm{n}^{\circ}$ 46467). Celui-ci ne mesure que 0,75/0,33 mm. Il est dans un état de conservation défectueux : la partie antérieure du Ver est racornie, avec bords latéraux ondulés (ce qui diminue notablement la longueur du corps). Contrairement à la description originale qui affirmait l'absence de ventouse ventrale, nous avons observé sans peine cet organe, partiellement recouvert par l'organe tribocytique en expansion; il mesure $40 / 42 \mu(6)$, tandis que la ventouse buccale atteint $50 / 55 \mu$. Le pharynx ellipsoïdal est très musculeux; il mesure $65 / 52 \mu$. L'œsophage $(30 / 20 \mu)$ a des parois plus épaisses que celles des caeca, mais la fixation défectueuse du Ver ne permet pas de distinguer la musculature circulaire. L'ovaire a $75 \mu$ de diamètre, le premier testicule, $125 / 85 \mu$ (le second est dilaté par compression). La poche du cirre, bien développée $(370 / 75 \mu$ ), s'étend jusqu'à mi-longueur du corps (qu'elle n'atteindrait pas si celui-ci était convenablement fixé) ; son extrémité proximale se trouve au niveau médian du testicule antérieur. Les œufs, au nombre de 2, mesurent 100 104/65-78 $\mu$ (l'un d'eux est dilaté) (7). Pour autant qu'on puisse le délimiter, le sphincter vaginal aurait comme dimensions $30 / 40 \mu$. Les huit spécimens recueillis par Chandler à Houston, Texas, proviennent de l'intestin grêle d'un chien.

Ces données comparatives permettent d'identifier le Mesostephanus longisaccus Chandler avec $M$. appendiculatus (Ciurea), dont la distribution géographique s'étend aux Etats-Unis (Price, 1928; Martin, 1961). Voir aussi : Malczewski (1964, p. 93 : tableau comparatif des deux taxons).

\section{Mesostephanus cubaensis Alegret, 1941}

Cette espèce cubaine provient de l'intestin de Phalacrocorax auritus floridanus (Audub.) (Surgidero de Batabanó, Prov. Habana). Le matériel original [dépôt à l'Université de Neuchâtel (G.D.), $\mathrm{n}^{\circ} \mathrm{N}$ 72-76] nous a été remis par I. Pérez Vigueras. C'est pourquoi nous pouvons compléter la description que Maria J. Alegret (1941, pp. 8-9, fig. 1) en a donnée.

Longueur du Ver 1,22-1,89 mm ; largeur 0,52-0,71 mm. Rapport L/1 = 2,43-2,94 ( 2,16 sur un exemplaire un peu contracté).

Corps longuement elliptique à lancéolé, légèrement concave ventralement, à appendice dorso-subterminal très réduit, à spinescence très fine s'étendant sur les faces

(5) Premier hôte intermédiaire: Cerithidea californica Haldeman; second hôte intermédiaire : Fundulus parvipinnis (Girard) et Gillichthys mirabilis Cooper. Adultes obtenus chez des poussins.

(6) Dubois (1951, p. 654 et fig. 4) avait déjà signalé l'existence d'une ventouse ventrale (diamètre 47 w.) chez un paratype de $M$. longisaccus.

(7) Chandler donne comme mesures 97-105/65-67 $\mu$. 
dorsale et ventrale jusqu'au niveau de l'acétabulum, plus en arrière sur les côtés (minuscules squamules cuticulaires disposées régulièrement en quinconce et ressemblant, en coupe optique, à des épines).

Ventouse buccale 65-90/50-75 $\mu$, plus petite que la ventouse ventrale 73-94/77-101 $\mu$, qui est située aux 45-53/100 de la longteur du corps. Pharynx ellipsoïdal ou arrondi, 47-68/ $37-60 \mu$, plus petit mais plus musculeux que les deux ventouses; œsophage moyen, 40-95 u. Organe tribocytique, 170-250/115-180 $\mu$ à l'état rétracté, s'ouvrant par une fente médiane.

Testicules ellipsoïdes, placés en tandem et en contiguïté, le premier 120230/160-265 $\mu$, le second toujours un peu plus grand, 170-255/200-310 $\mu$. Ovaire 85-120/90-140 $\mu$, situé latéralement dans la zone du testicule antérieur et opposé à la poche du cirre longuement claviforme, 520-740/65-85 u., qui atteint ou dépasse généralement le bord frontal de ce testicule ; diamètre du cirre 14-17 ‥ Sphincter vaginal 20-40/3550 u. Follicules vitellogènes disposés autour de l'organe tribocytique en une couronne dont le diamètre moyen varie de 350 à $500 \mu$, et dont le front se situe aux 45-56/100 de la longueur du corps; rapport de cette longueur à ce diamètre moyen $=3,5-3,9(2,1$ pour un exemplaire un peu contracté, 4 pour un autre en extension). Eufs 96-110/68-73 $\mu$, au nombre de 2 à 15 .

Le spécimen représenté par la figure 1 est désigné comme lectotype [Université de Neuchâtel (G.D.), $\mathrm{n}^{\circ}$ N 74].

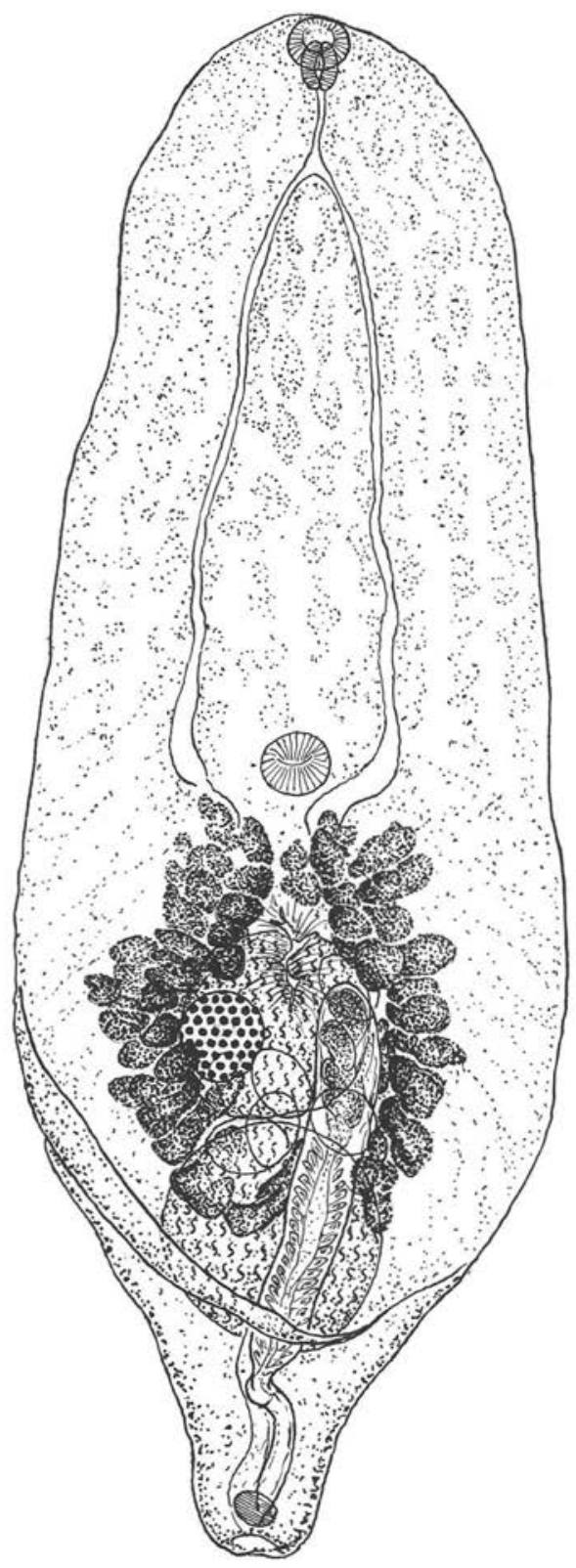

FIG. 1. - Mesostephanus cubaensis Alegret, de Phalacrocorax auritus floridanus (Audub.). Lectotype : $1,89 \mathrm{~mm}$. Vue ventrale. Univ. Neuchâtel (G.D.), $\mathrm{n}^{\circ} \mathrm{N} 74$. 
Mesostephanus cubaensis se rapproche de deux autres espèces néotropicales à ventouse ventrale plus grande que la ventouse buccale:

M. odhneri (Travassos, 1924) Lutz, 1935 (Brésil, Porto Rico), de Sula sula (L.) et de Fregata aquila (L.) (cf. Dubois, 1970, pp. 190-193, fig. 1 et 2) (8);

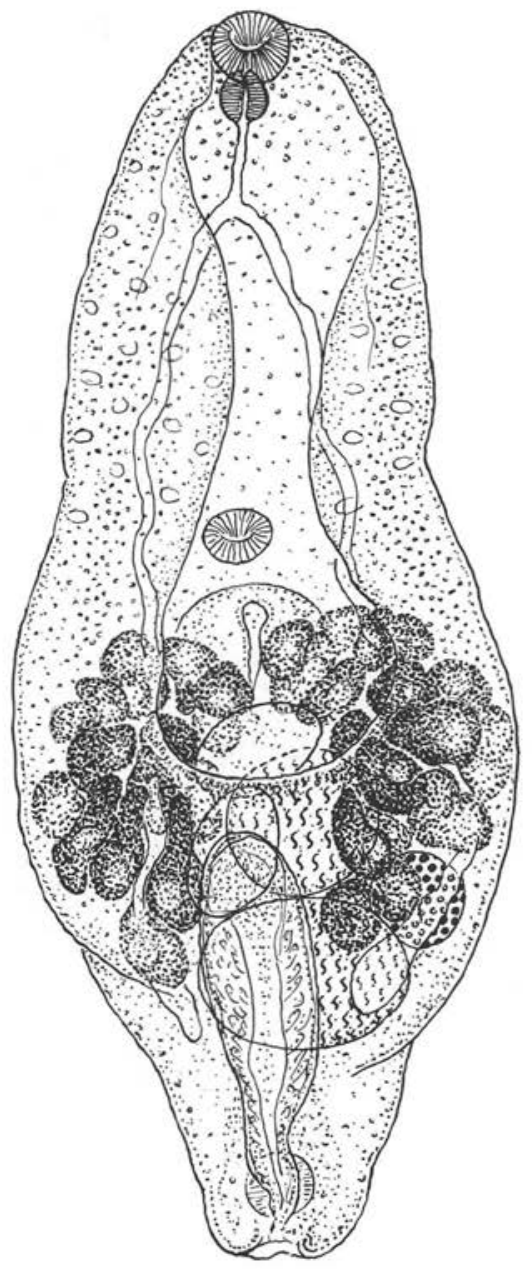

FIG. 2. - Mesostephanus dottrensi Baer, de Scotopelia peli Bonap. Syntype : $0,79 \mathrm{~mm}$. Vue ventrale. Univ. Neuchâtel (J.-G. B.).

M. microbursa Caballero, Grocott et Zerecero, 1953 (Panama et île de Coronado, en Basse-Californie, Floride), de Pelecanus occidentalis californicus Ridgway (cf. Caballero, Grocott et Zerecero, 1953, pp. 398-402, fig. 5 et 6 ; Dubois, 1958 , pp. $74-76$, fig. 4 et 5).

$M$. odhneri se distingue de $M$. cubaensis par la situation plus antérieure de la ventouse ventrale (aux 30-45/100 de la longueur du corps) et par le plus grand développement de la couronne vitelline (rapport: longueur du Ver/diamètre moyen de cette couronne = 2-3).

M. microbursa s'oppose à $M$. cubaensis également par la situation plus avancée de l'acetabulum (aux 32-42/100), par le plus grand développement de l'appendice dorsosubterminal et par la forme plus trapue de la poche du cirre (dont la pars prostatica occupe la majeure partie) qui n'atteint que le bord frontal du second testicule ou le niveau intertesticulaire (ceci en rapport avec le second des caractères discriminants).

\section{Mesostephanus dottrensi Baer, 1957}

Syn. Mesostephanus crociduri Mikhail et Fahmy, 1968.

J.-G. Baer (1957, pp. 560-563, fig. 8-9) avait décrit son $M$. dottrensi d'après une virgztaine d'exemplaires recueillis dans le premier tiers de l'intestin d'une chouette

(8) M. odhneri, décrit de Nyctanassa violacea (L.), a encore été retrouvé chez un Ardéidé, Tigrisoma lineatum marmoratum (Vieill.) (cf. Dubois, 1970, p. 191). 
pêcheuse, Scotopelia peli Bonap. (hôte accidentel), tirée au bord du Marahoué, dans la région de Mankono, en Côte-d'Ivoire.

J. W. Mikhail et M. A. M. Fahmy (1968, pp. 424-426, fig. 2) obtenaient leur M. crociduri de 10 (sur 30) Crocidura olivieri (Lesson) (9), hôte inusuel, provenant de El-Faiyum et de Miet Fadali, en Egypte.

Les deux taxons nous paraissent identiques; ils présentent en commun les caractères suivants (fig. 2) :

1) corps typiquement spathacé, de petite taille $(<1 \mathrm{~mm})$;

2) ventouse ventrale un peu moins grande que la ventouse buccale; pharynx plus petit que cette dernière ;

3) ovaire submédian à latéral, situé au niveau intertesticulaire ;

4) poche du cirre s'allongeant jusque dans la zone du testicule antérieur.

Les mesures données par les auteurs sont concordantes:

\begin{tabular}{|c|c|c|}
\hline & dottrensi & crociduri \\
\hline Longueur du corps $\ldots \ldots \ldots \ldots$ & $0,68-0,78 \mathrm{~mm}$ & $0,63-0,75 \mathrm{~mm}$ \\
\hline Largeur $\ldots \ldots \ldots \ldots \ldots \ldots$ & $0,31-0,33$ & $0,30-0,42$ \\
\hline $\begin{array}{l}\text { Ventouse buccal } \ldots \ldots \ldots \ldots \\
\text { Pharynx } \ldots \ldots \ldots \ldots \ldots \ldots \\
\text { Ventouse ventrale } \ldots \ldots \ldots \ldots\end{array}$ & $\begin{array}{r}45-48 \mu \\
45 / 30 \\
45\end{array}$ & $\begin{array}{l}45-54 / 30-55 \mu \\
30-40 / 31-43 \\
30-40 / 41-44\end{array}$ \\
\hline Ovaire $\ldots \ldots \ldots \ldots \ldots \ldots \ldots$ & 90 & $90-110 / 60-70$ \\
\hline Testicule antérieur $\ldots \ldots \ldots \ldots$. & 102 & $120-190 / 110-190$ \\
\hline Testicule postérieur ......... & $136 / 91$ & $120-150 / 100-170$ \\
\hline Poche du cirre $\ldots \ldots \ldots \ldots \ldots$ & $227 / 27$ & $280-430 / 50-70(10)$ \\
\hline Eufs $\ldots \ldots \ldots \ldots \ldots \ldots \ldots$ & $91 / 59$ & $83-100 / 58-68$ \\
\hline Nombre d'œufs $\ldots \ldots \ldots \ldots \ldots$ & jusqu'à 3 ou 4 & 1 à 4 \\
\hline
\end{tabular}

L'examen du matériel type de $M$. dottrensi nous permet de préciser quelques traits et de compléter les mesures données par Baer :

Longueur/largeur du Ver: 0,64-0,95/0,27-0,38 mm. Un spécimen ovigère (5 œufs) mesure à l'état contracté $0,69 / 0,39 \mathrm{~mm}$ : c'est celui que représente la figure 8 (à droite) de la description originale.

\begin{tabular}{|c|c|}
\hline Ventouse buccale & $50-55 / 52-57 \mu$ \\
\hline Pharynx $\ldots \ldots \ldots \ldots \ldots \ldots \ldots \ldots \ldots$ & $40-47 / 34-37$ \\
\hline Ventouse ventrale $\ldots \ldots \ldots \ldots \ldots \ldots \ldots$ & $37-52 / 45-55$ \\
\hline CEsophage & $25-50$ \\
\hline
\end{tabular}

(9) Nous n'avons pas reçu en prêt le matériel type de $M$. crociduri malgré des demandes adressées au $\mathrm{P}^{r}$ M. A. M. Fabmy et au Parasitology Department, Namru-3, au Caire.

(10) La longueur de la poche du cirre, mesurée sur la figure 2, n'excède pas $300 u$. Elle ne saurait atteindre plus de la moitié de la longueur du corps. 


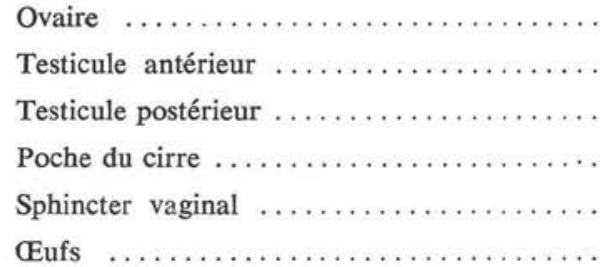

Nombre d'œufs
68-75/80-95

105-125/120-145

$100-140 / 120-180$

250-365/50-70

40-55

85-92/57-59

1 à 5 (le plus souvent 2 ou 3)

Le revêtement de squamules cuticulaires ne s'étend guère au-delà de l'organe tribocytique. Les glandes dorsales sont disposées en une quinzaine de rangées longitudinales plus ou moins régulières (visibles surtout sur le spécimen contracté) (cf. Baer, fig. 8 , à droite).

La ventouse buccale est toujours un peu plus grande que l'acetabulum faiblement musculeux et situé entre les $41-47 / 100$ de la longueur du corps. Le pharynx est plus petit que les ventouses. Les caeca s'allongent jusqu'au niveau de la seconde moitié ou du bord postérieur du second testicule.

\section{Mesostephanus baliasturis Tubangui et Masiluñgan, 1941}

Syn. Mesostephanus minor Dubois et Pearson, 1965.

La mise en synonymie de $M$. minor (Queensland: Australie) avec $M$. haliasturis (Luzon : Philippines) a été jugée admissible par Dubois et Angel (1972, p. 214). La description originale était basée sur un matériel provenant de Haliastur intermedius Blyth. Dubois et Pearson (1965) obtenaient M. minor de Haliastur sphenurus (Vieill.). Ils le retrouvaient (1967) chez un Pelecanus conspicillatus Temm. et chez une Anhinga novaehollandiae (Gould). Enfin, Dubois et Angel (op. cit.) redécrivaient l'espèce sous le nom de haliasturis, en rectifiant l'appellation originale (haliasturus), d'après un nouveau matériel recueilli chez Pelecanus conspicillatus qui paraît être un des hôtes normaux.

Mesostephanus haliasturis se caractérise par sa région antérieure élargie, à «épaules » bien marquées, d'où les bords latéraux du corps se replient ventralement pour se réunir postérieurement au-devant de l'appendice dorso-subterminal courtement tronconique. Les vitellogènes n'atteignent pas le niveau de la ventouse ventrale (distance 28 à $35 \mu$ ), celle-ci étant un peu plus grande que la ventouse buccale. La poche du cirre peut s'allonger jusqu'à la hauteur du premier testicule ou même au-delà (fig. 3 ). 


\section{Mesostephanus microbursa Caballero, Grocott et Zerecero, 1953}

Cette espèce, qui peut atteindre $2 \mathrm{~mm}$ de longueur, a comme caractères nettement distinctifs un appendice dorso-subterminal relativement grand, long de 360 à $550 \mu$, mesurant 260 à $380 \mu$ à sa base et 140 à $210 \mu$ au sommet, et une poche du cirre massivement claviforme, n'atteignant que la zone du testicule postérieur (ou tout au plus l'espace intertesticulaire), large de 130 à $170 \mu$ au niveau de la pars prostatica bien développée, et dont la vésicule séminale n'occupe en moyenne que le cinquième de l'organe. $\mathrm{La}$ ventouse ventrale (47-78/51-89 $\mu$ ), située au 32-42/100 de la longueur du Ver, est à peine plus grande que la ventouse buccale (45-68/45-68 $\mu$ ) (11), mais elle est faiblement musculeuse, «muy poco perceptible», parfois soulevée par un bourrelet de la surface du corps. L'ovaire se situe au niveau de la seconde moitié du testicule antérieur. Le sphincter vaginal est très faiblement musculeux et, par conséquent, difficile à observer et à délimiter (fig. 4).

Décrite par Caballero, Grocott et Zerecero (1953) comme parasite de Pelecanus occidentalis californicus Ridway, en provenance de la ville de Panama (Amérique centrale) et de l'île de Coronado (Basse Californie, Mexique), l'espèce avait déjà été récoltée par June Mahon, en 1940, dans le même hôte et la même île, et redécrite par Dubois (1958, pp. 74-76, fig. 4-5). Elle fut encore retrouvée par Charles H. Courtney, M. S. Cand. de l'University of Florida, Gainesville, à Bird Key, Lee Co., Fla., le 23 mai 1972, toujours chez le Pélican brun [préparation $\mathrm{n}^{\circ}$ W 3 (G.D.), dans la collection de l'Université de Neuchâtel].

(11) Cependant, Caballero et al. représentent (fig. 5) un exemplaire dont l'acetabulum est plus petit que la ventouse buccale. Chez des spécimens longs de 1,9 à $2 \mathrm{~mm}$, recueillis par Charles $\mathrm{H}$. Courtney, en Floride (vide infra), le diametre de la ventouse buccale est de 80-95/105-110 u, celui de la ventouse ventrale, très faiblement musculeuse, de 80-90/90-120 u. 


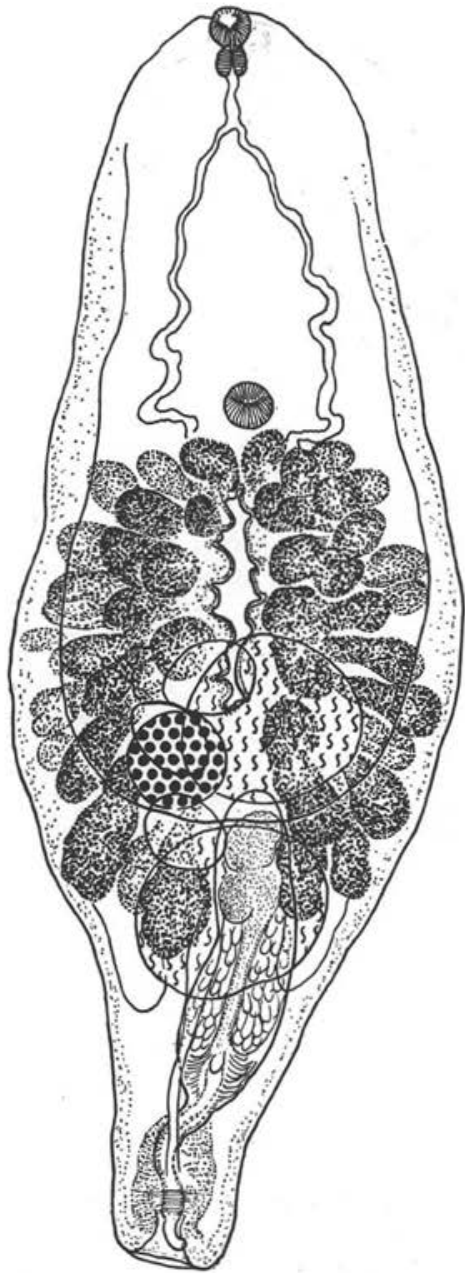

FIG. 4. - Mesostephanus microbursa Caballero, Grocott et Zerecero, de Pelecanus occidentalis californicus Ridgway. Longueur: $1,35 \mathrm{~mm}$. Vue ventrale. Univ. Neuchâtel (G.D.), n ${ }^{\circ}$ O 69 (June Mahon leg.).
G. DUBOIS

\section{Mesostephanus odbneri (Travassos, 1924) Lutz, 1935}

Syn. Mesostephanus appendiculatoides Cable, Conner et Balling, 1960, nec Price, 1934.

Autres synonymes: cf. Dubois, 1970, pp. 190-191.

Cette espèce brésilienne, décrite par Travassos d'après de nombreux spécimens obtenus expérimentalement dans l'intestin de quelques «Socos » [Nyctanassa violacea (L.)], a été retrouvée plusieurs fois chez Sula sula (L.) et Fregata aquila (L.), une seule fois chez Tigrisoma lineatum marmoratum (Vieill.) dans la collection A. Lutz de l'Institut Oswaldo Cruz, Rio de Janeiro, et redécrite par Dubois (1970). Cable, Connor et Balling (1970) l'ont confondue avec Mesostephanus appendiculatoides (Price) (vide supra: texte relatif à cette espèce).

Le spécimen déposé par ces auteurs à l'U.S. National Museum (Helm. coll. $\mathrm{n}^{\circ}$ 38207) (12) et représenté par la figure 5 de leur travail provient de Sula leucogaster (Bodd.) (Porto Rico). Il est bien caractéristique de $M$. odhneri.

Longueur $0,76 \mathrm{~mm}$, largeur $0,39 \mathrm{~mm}$. Ventouse buccale $47 / 50 \mu$; ventouse $50 / 57 \mu$, située aux $38 / 100$ de la longueur du corps; pharynx 42/29 $\mu$; œsophage $52 \mu$. Organe tribocytique rétracté $120 / 110 \mu$. Ovaire 76/68 $\mu$, localisé latéralement au niveau du testicule antérieur et opposé à la poche du cirre longuement claviforme, 400/57 $\mu$, dont l'extrémité libre se recourbe au-devant de cette glande mâle. Follicules vitellogènes disposés en une couronne dont le front atteint le bord postérieur de la ventouse ventrale. Testicule antérieur $90 / 120 \mu$, testicule postérieur 105/130 $\mu$. CEufs (au nombre de 4) 90-100/65-69 $\mu$ (13). Rapport longueur corps/diamètre moyen couronne vitelline $=2,4$.

(12) Ce spécimen se trouve parmi quatre exemplaires de Mesostephanus fajaráensis (Price) provenant de Sula leucogaster (15-VI-1952).

(13) Cable et al. donnent comme dimensions 102-111/73-84 u. ; elles correspondent à nos mesures (1970, p. 193). 


\section{Mesostephanus sknorzowi Petrov, 1950}

Syn. Mesostephanus sp. Malczewski, 1962 ;

Mesostephanus alopicis Malczewski, 1964.

On ne saurait guère être dans le doute au sujet de la mise en synonymie de M. alopicis (Pologne) avec M. skworzowi (U.R.S.S.). Les deux taxons, l'un provenant du Renard polaire (Alopex lagopus L.), l'autre du Chat (Felis domestica L.), sont de faible taille $(0,52-0,95 \mathrm{~mm})$, avec un appendice dorso-subterminal très réduit $(0,027$ $0,060 \mathrm{~mm}$ de long sur 0,050-0,108 $\mathrm{mm}$ de diamètre à la base, pour alopicis). Ils ont tous deux un pharynx très musculeux $(33-62 / 37-73 \mu$ ), presque aussi gros ou même plus gros (par dilatation) que la ventouse buccale, et un acetabulum très petit (22-38/ 20-45 $\mu$ ), situé à mi-longueur du corps ou à peine plus en avant. Les œufs ont de grandes dimensions (177-120/70-73 $\mu$ pour skworzowi, 105-150/53-117 $\mu$ pour alopicis). Le sphincter vaginal est petit, faiblement développé ; Petrov ne l'a pas observé, si bien que Sudarikov in Skrjabin (1961, p. 380, fig. 144) a cru devoir transférer le Mesostephanus skworzowi dans le genre Paracoenogonimus Katsurada.

\section{Nouvelle diagnose du genre Mesostephanus Lutz, 1935}

Cyathocotyloidea: Prohemistomoinea: Prohemistomidae: Prohemistominae à corps ovale ou linguiforme, foliiforme ou cochléariforme, parfois spathacé, prolongé par un petit appendice dorso-subterminal à l'extrémité duquel s'ouvre le pore génital ; à organe tribocytique protrusible, petit ou moyen, avec fente médiane, confiné en arrière de la ventouse ventrale qui est toujours présente, quoique souvent faiblement musculeuse, et située environ à mi-longueur du corps; à testicules subglobulaires à ovoïdes, placés en tandem; à ovaire submédian ou latéral, localisé au niveau intertesticulaire ou dans la zone du testicule antérieur et opposé à la poche du cirre bien développée ; à sphincter vaginal ellipsoïde, plus ou moins apparent mais toujours présent ; à follicules vitellogènes disposés en couronne excentrée par rapport à l'organe tribocytique et ne dépassant pas le niveau acétabulaire (14).

Cercaires se développant dans des Mollusques Cérithiidés. Métacercaires hébergées par des Poissons. Adultes parasites de Pélécaniformes, occasionnellement de Rapaces (Milans, Haliastur, Chouette pêcheuse), de Carnivores (Chien, Renard, Chat) ou d'Insectivores (Musaraignes).

Espèce type : Mesostephanus fajardensis (Price, 1934) Lutz, 1935.

(14) D'où l'appellation générique par opposition à Prosostephanus Lutz, genre dans lequel cette couronne (stephanos) se développe jusqu'au voisinage du pharynx (cf. Lutz, 1935, p. 166 ou p. 179). 


\section{Bibliographie}

Alegret (M. J.), 1941. - Contribución al conocimiento de los vermes parásitos de Phalacrocorax Auritus Floridanus, La Habana, 32 p.

BAER (J.G.), 1957. - Trématodes et Cestodes récoltés en Côte d'Ivoire, avec remarques sur la famille des Dicrocoeliidae Odhner et sur les parasites des Damans. Rev. suisse Zool., 64, 547-575.

Caballero (E.), Grocott (R. G.) et Zerecero (M. C.), 1953. - Helmintos de la Republica de Panama. IX. Algunos trematodos de aves marinas del Oceano Pacifico del Norte. An. Inst. Biol. Méx., 24, 391-414.

Cable (R. M.), Connor (R. S.) et Balling (J.W.), 1960. - Digenetic trematodes of Puerto Rican shore birds. Sci. Surv. P. R., 17, 191-255.

Chander (A. C.), 1950. - Mesostephanus longisaccus, a new cyathocotylid trematode from a dog. J. Parasit., 36, 90.

CIUREA (I.), 1916. - Prohemistomum appendiculatum, eine neue Holostomiden-Art aus Hunden- und Katzendarm, dessen Infektionsquelle in den Süsswasserfischen zu suchen ist. Nebst einer Bemerkung zu der Arbeit Prof. Katsurada «Studien über Trematodenlarven bei Süsswasserfischen, mit besonderer Berücksichtigung der Elbund Alsterfische ». Z. Infektkr. Berlin, 17, 309-328.

DenNis (E. A.), 1968. - Biological studies on the life history of Mesostephanus yedeae sp. n. (Trematoda: Cyathocotylidae). Diss. Abstr., 28 B, 3523-3524.

DuboIs (G.), 1938. - Monographie des Strigeida (Trematoda), Mem. Soc, neuchâtel. Sci. nat., $6,1-535$.

DuBors (G.), 1951. - Nouvelle clé de détermination des groupes systématiques et des genres de Strigeida Poche (Trematoda). Rev. suisse Zool., 58, 639-691.

Dubors (G.), 1958. - Les Strigeida (Trematoda) de Californie de la collection June Mahon. Bull. Soc. neuchâtel. Sci. nat., 81, 69-78.

DuboIs (G.), 1970. - Les Strigeata (Trematoda) de la collection A. Lutz. Mem. Inst. Oswaldo Cruz, 68, 169-196.

Dubois (G.) et ANGel (L. M.), 1972. - Strigeata (Trematoda) of Australian birds and mammals from the helminthological collection of the University of Adelaide. Trans. R. Soc. S. Aust., 96, 197-215.

Dubois (G.) et Pearson (J. C.), 1963. - Les Strigeida (Trematoda) d'Egypte (collection William H. Wells). Ann. Parasit. hum. comp., 38, 77-91.

Dubois (G.) et Pearson (J. C.), 1965. - Quelques Strigeida (Trematoda) d'Australie. Bull. Soc. neuchâtel. Sci. nat., 88, 77-99.

Dubois (G.) et Pearson (J. C.), 1967. — Ibid., II. Ibid., 90, 185-204.

Hutron (R.F.), 1964. - A second list of parasites from marine and coastal animals of Florida. Trans. Amer. micr. Soc., 83, 439-447.

Hutton (R. F.) et Sogandares-Bernal (F.), $1960 a$. - Studies on helminth parasites from the coast of Florida. II. Digenetic trematodes from shore birds of the west coast of Florida. 1. Bull. Mar. Sci. Gulf and Caribbean, 10, 40-54. 
Hutton (R. F.) et Sogandares-Bernal (F.), 1960 b. - Preliminary notes on the life-history of Mesostephanus appendiculatoides (Price, 1934) Lutz, 1935. Ibid., 10, 234-236.

Kuntz (R. E.) et Chandler (A. C.), 1956. - Studies on Egyptian trematodes with special reference to the Heterophyids of mammals. I. Adult flukes, with descriptions of Phagicola longicollis n. sp., Cynodiplostomum namrui n. sp., and a Stephanoprora from cats. J. Parasit., 42, 445-459.

LuTZ (A.), 1935. - Observações e considerações sobre Cyathocotylineas e Prohemistomineas. Mem. Inst. Osw. Cruz., Rio de J., 30, 157-168 (trad. allem., 169-182).

MALCZEwsKi (A.), 1962. - Helminth parasites of bred foxes and minks in Poland. Acta Parasit. polon., 10, 231-260.

Malczewski (A.), 1964. - Mesostephanus alopicis sp. n. Bull. Acad. polon. Sci., Cl. 2, 12, 91-94.

MARTIN (W.E.), 1961. - Life cycle of Mesostephanus appendiculatus (Ciurea, 1916) Lutz, 1935 (Trematoda : Cyathocotylidae). Pacif. Sci., 15, 278-281.

Mikhail (J. W.) et FaHMY (M. A. M.), 1968. - Two new species of trematodes from insectivores. Zool. Anz., 181, 421-426.

Petrov (A. M.), 1950. - [Nouveau trématode de l'intestin du chat, Mesostephanus skworzowi nov. sp. (Strigeata)]. Trudi Vsesoy. Inst. Gelmint. Akad. K. I. Skrjabin, 4, 81-82.

PRICE (E. W.), 1928. - The occurrence of Prohemistomum appendiculatum in the United States. J. Parasit., 15, 68.

Price (E. W.), 1934. - New trematodes parasites of birds. Smithson. Misc. Coll., 91, 1-6.

Sudarikov (V. E.), in SKrJabin (K. I.), 1961. - [Trématodes des animaux et de l'homme. Eléments de trématodologie]. Acad. Sci. U.R.S.S. 19, 267-471.

Travassos (L.), 1924. - Nouvelle espèce de Prohemistomum. C.R. Soc. Biol. (Sect. brés.), 91, 835-838.

Tubangui (M. A.) et Masilungan (V. A.), 1941. - Trematode parasites of Philippine vertebrates. IX. Flukes from the domestic fowl and other birds. Philipp. J. Sci., 75, $131-142$. 